\title{
Early Decannulation, Repatriation, and Hospital Discharges by Introducing a Tracheostomy Care Task Force for Noncritical Care Tracheostomized Patients
}

\author{
Raees Ahmed, ${ }^{1}$ Syed Tabish R. Zaidi, ${ }^{2}$ Hasan Husein Hasan Moshtohry, ${ }^{1}$ Khalil Ahmad, ${ }^{3}$ \\ Younis Ameen Kazim, ${ }^{4}$ Amani Adnan Charaf, ${ }^{5}$ and Jawed Abubaker, ${ }^{1}$ \\ ${ }^{1}$ Department of Medical Intensive Care Unit, Rashid Hospital Trauma Center, P.O. Box 4545, Dubai, United Arab Emirates \\ ${ }^{2}$ Department of Pharmaceutical Care, King Abdulaziz. Medical City, Al Ahsa 31982, Saudi Arabia \\ ${ }^{3}$ Department of Surgical Intensive Care Unit, Rashid Hospital Trauma Center, P.O. Box 4545, Dubai, United Arab Emirates \\ ${ }^{4}$ Director Medical Affairs, Rashid Hospital Trauma Center, P.O. Box 4545, Dubai, United Arab Emirates \\ ${ }^{5}$ Department of Nursing Neurosurgical Unit, Rashid Hospital Trauma Center, P.O. Box 4545, Dubai, United Arab Emirates \\ Address correspondence to Raees Ahmed, ahmedraees@hotmail.com
}

Received 26 December 2011; Revised 19 June 2012, Accepted 30 June 2012

\begin{abstract}
Objective. To determine the impact of an intensivist lead tracheostomy care team on the number of successful decannulations in non-critically ill patients and the time required for such decannulations and discharge from the hospital. Methods. Following the introduction of a multidisciplinary tracheostomy care task force, data was collected prospectively from July to December 2009. Matching control data was collected retrospectively from January to June 2009. Chi-Square and Mann-Whitney Utest were used to compare the differences in study variables with an $\alpha$ of 0.05 . Results. A total of 44 and 47 patients with percutaneous tracheostomy (PCT) were discharged from the ICU pre- and post-implementation of the task force, respectively. Nine patients in pre- and 24 in postimplementation phase were decannulated, discharged from the hospital, and repatriated to their country of origin (Chi Sq. $=9.21, P=.002$ ). Patients in pre-implementation group had longer hospital stay post ICU discharge compared to patients in post-implementation (58 vs. 34.1 days) group $(P<.001)$. Conclusion. Structured multidisciplinary tracheostomy care team was associated with significant increase in number of decannulations, hospital discharges, and repatriation post ICU discharge.
\end{abstract}

Keywords decannulations; protocol; taskforce; tracheostomy care team; United Arab Emirates; trauma; early discharge and repatriation; non-critically ill

\section{Introduction}

Insertion of percutaneous tracheostomy (PCT) has become a standard procedure usually performed by the intensivist on the critically ill patients. Post procedure, the tracheostomy care is mostly provided by the intensive care unit (ICU) nurses and ICU physicians. As there are no specialized tracheostomy teams available in many institutions, post ICU discharge the routine tracheostomy care is usually done by the ward nurses and respiratory therapist, without any close follow up by the operating team. This gap in continuity of care may lead to the delivery of suboptimal care to this group of patients.

In most of the developed countries, there are both post-acute and long-term facilities, where such patients get optimum tracheostomy care and possible weaning and decannulation. In countries like United Arab Emirates (UAE) and especially in Dubai, where post-acute care facilities and rehabilitation centers are not available, most of these patients stay in an acute care facility until they are discharged. This further complicates the situation as the majority of these patients are non-United Arab Emirates (UAE) nationals, that is, they are expatriates and they need to be transferred back to their country of origin (repatriation). Thus, the discharge and repatriation process gets delayed further as the tracheostomized patients cannot be sent directly to their home in the country of origin and they have to be transferred to a hospital in their home place. Furthermore, for the safety of the patient and as per most of these commercial airlines policy, these tracheostomized patients need to be escorted with a nurse and a physician, which tremendously increases the cost of traveling, which has to be borne by the family of the patients.

\section{Background}

Rashid Hospital Trauma Center is the largest 600-bed trauma and tertiary referral center of the United Arab Emirates, with an average emergency visit of 175,000200,000 per year. The Medical Intensive Care Unit has a capacity of 30 beds, which receives 900 to 1000 admissions 


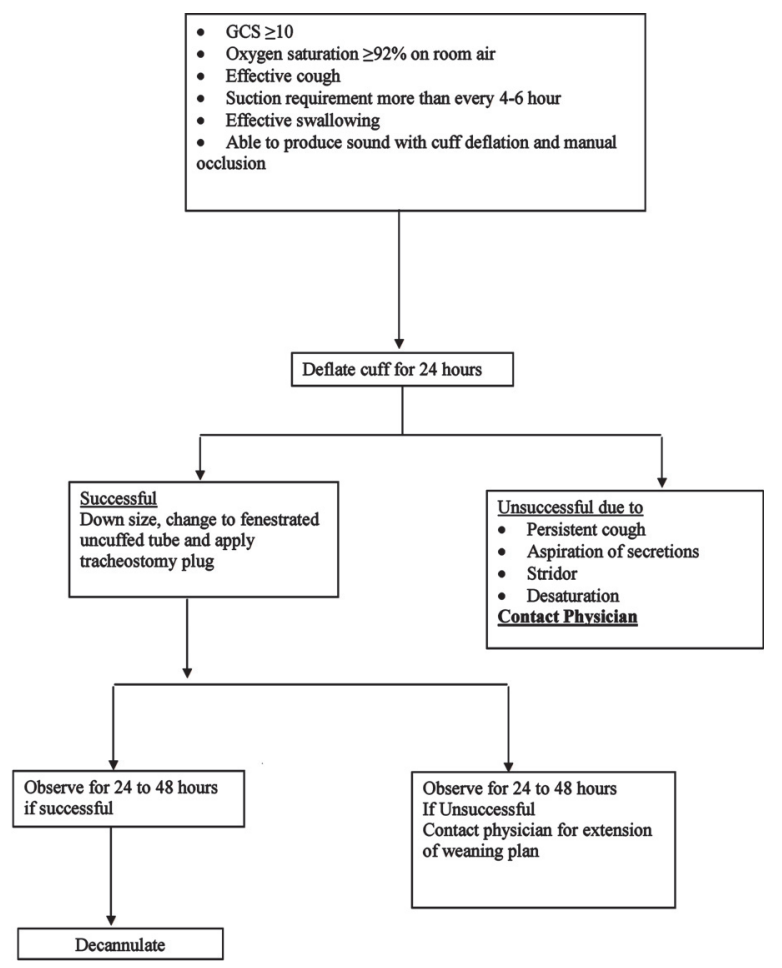

Figure 1: Protocol used for weaning and decannulation in pre- and post-implementation phases of the study.

per year. A large number of patients admitted under trauma category have severe traumatic brain injury which is one of the common indications for admission to ICU. An average of approximately 80 to 100 patients per year require a tracheostomy and percutaneous tracheostomy is the preferred procedure in our ICU. Post ICU discharge, these tracheostomized patients are followed by the intensivist for a routine change of tracheostomy tube, which according to the hospital policy is 60 days from time of insertion or when the cuff or tube patency gets problematic. Beside a routine change of tracheostomy tube, the treating physician or the charge nurse along with respiratory therapist assess patients for weaning and decannulation screening criteria, as per weaning and decannulation protocol of Rashid Hospital (Figure 1). The respiratory therapist coordinates with intensivist to further assess patients for possible weaning and decannulation.

Due to a continuous shortage of critical care beds along with delayed hospital discharges of tracheostomized patients from the hospital, the Quality Department of Rashid Hospital along with the hospital administration decided to introduce an intensivist lead tracheostomy care taskforce (TCT). The members of the task force were an intensivist, respiratory therapist, and ward charge nurse. The patients were followed by the task force on a biweekly basis and identified suitable patients who could be safely decannulated as per the protocol.
The primary aim of this study was to determine whether an intensivist lead TCT can increase the number of tracheostomized patients who can be successfully decannulated. The secondary aim was to determine if an intensivist lead TCT can decrease the time required for the successful decannulation of tracheostomized patient and discharge from the hospital as well as subsequent repatriation to their country of origin.

\section{Material and methods}

The tracheostomy care taskforce (TCT) was established in July 2009. The prospective arm of this study comprises all the patients discharged from the ICU with a tracheostomy tube in situ during July-December 2009. The retrospective control arm of this study comprises all the patients discharged from the ICU with a tracheostomy tube in situ during January-June 2009. The data on the following study variables was collected: age, gender, admitting diagnosis, indication for tracheostomy, total number of days of tracheostomy tube being in place from the time of insertion, Glasgow coma scale at the time of decannulation, total number of decannulations, total number of patients who failed weaning and decannulation, any medical reason of failure of weaning and decannulation, number of patients requiring intubation post decannulation, number of patients requiring ICU admission post decannulation, number of days from ICU discharge up to decannulation, number of days post decannulation up to hospital discharge, and number of patients who were discharged from the hospital with tracheostomy tube in place. Weaning failure was defined as when patient cannot tolerate uncuffed, fenestrated tube with tracheostomy plug, due to stridor, hypoxia, respiratory distress, difficulty in mobilizing secretions, and a weak cough reflex.

Descriptive statistics was computed for all the study variables. Since the primary outcome measures were binary in nature, normal distribution pattern was computed for secondary outcome measure to determine the appropriate inferential statistics. (Kolmogorov-Smirnova test for normality with an $\alpha=.01$ for both variables.) We decided to use Chi-Square test to examine the difference between primary outcome measures depending on the normality distribution test, either independent Student's t-test (if normal distribution) or Mann-Whitney U test (if non-normal distribution) for secondary variables. All analyses were performed using SPSS Version 20.0 (SPSS Inc., Chicago, IL, USA). The Ethical and Research committee of Rashid Hospital approved the project and waived the requirement of informed consent, as it was regarded as a quality project.

\section{Results}

Seven hundred and twenty five patients were discharged from the Medical ICU in the year 2009. A total of 91 
Table 1: Baseline characteristics and study outcome measures.

\begin{tabular}{|c|c|c|c|}
\hline Variables & Pre-taskforce $(n=44)$ & Post-taskforce $(n=47)$ & $\operatorname{Sig}(\alpha=.05)$ \\
\hline Mean age & $35 \pm 10.1$ & $35.8 \pm 10.3$ & NS \\
\hline Male (\%) & $38(86)$ & $37(78)$ & NA \\
\hline Severe traumatic brain injury $(\%)^{*}$ & $24(54)$ & $25(53)$ & NS \\
\hline Severe cerebral hemorrhage $(\%)^{*}$ & $11(25)$ & $13(27)$ & NS \\
\hline Ischemic stroke with surgical intervention $(\%)^{*}$ & $6(14)$ & $5(11)$ & NS \\
\hline Mean GCS of all patients & $9.04 \pm 1.3$ & $9.65 \pm 2.0$ & NS \\
\hline Mean GCS of decannulated patients & $11.3 \pm 0.45$ & $11.5 \pm 0.5$ & NS \\
\hline Non-UAE nationals $(\%)$ & $41(95 \%)$ & $42(89 \%)$ & NS \\
\hline Mean days from tube insertion to ICU discharge & $5.1 \pm 1.6$ & $5.8 \pm 0.9$ & NS \\
\hline Mean days of tube in situ post ICU discharge & $49 \pm 8.6$ & $30 \pm 4.1$ & $P<.001^{\#}$ \\
\hline Mean total days of tube in situ & $56.4 \pm 11.3$ & $36.3 \pm 3.5$ & $P<.001^{\#}$ \\
\hline Mean days post decannulation to discharge & $4.0 \pm 1.0$ & $4.2 \pm 0.8$ & NS \\
\hline Mean days of hospital stay post ICU discharge & $58.0 \pm 11.3$ & $34.1 \pm 3.6$ & $P<.001^{\#}$ \\
\hline Total decannulations $(\%)$ & $9(20)$ & $24(51)$ & $P=.02^{\sim}$ \\
\hline Number died with tube in situ $(\%)$ & $2(4)$ & $4(8)$ & NS \\
\hline Number discharge with tube in situ (\%) & $3(7)$ & $5(11)$ & NS \\
\hline Number of patients who failed decannulation (\%) & $35(80)$ & $23(49)$ & NS \\
\hline Number with sub glottis stenosis $(\%)$ & $2(4)$ & $1(2)$ & NS \\
\hline
\end{tabular}

${ }^{*}$ Not mutually exclusive so may not add up to $100 \%$.

$\mathrm{NS}=$ not significant; ${ }^{\#}$ Mann-Whitney U test; $~{ }^{\sim}$ Chi-Square test.

patients had a tracheostomy tube in place at the time of ICU discharge; none of these patients required a ventilator support and were on a maximum of $30 \%$ fraction of inspired oxygen via a thermal humidifier. Forty-four patients were discharged in the pre-implementation phase whereas 47 patients during the post-implementation phase; baseline characteristics of both groups as well as study outcome measures have been summarized in Table 1. The commonest indication for PCT was severe traumatic brain injury followed by spontaneous cerebral hemorrhage and Ischemic stroke with surgical intervention. There were no significant differences in any of the baseline characteristics between the two groups of patients (Table 1).

The mean number of days from tracheostomy insertion to ICU discharge was comparable in both groups (5.2 \pm 1.6 vs. $5.8 \pm 0.92$ ). The mean number of days patients took to be decannulated was reduced from $49 \pm 8.67$ days in pre-implementation phase to $30 \pm 4.17$ days in the post-implementation phase $(P<.001)$; Figure 2 displays the median number of days as well as the respective interquartile ranges. Nine $(20 \%)$ patients in pre- and 24 $(51 \%)$ in post-implementation phase were successfully decannulated (Chi Sq. $=9.21, P=.002$ ); all decannulated patients were subsequently discharged from the hospital as well as repatriated to their country of origin. Three (7\%) patients in pre-implementation and $5(11 \%)$ patients in post-implementation phase were UAE nationals and were discharged to go home with tracheostomy tube in place on their family request prior to decannulation. The length of hospital stay post ICU discharge was significantly

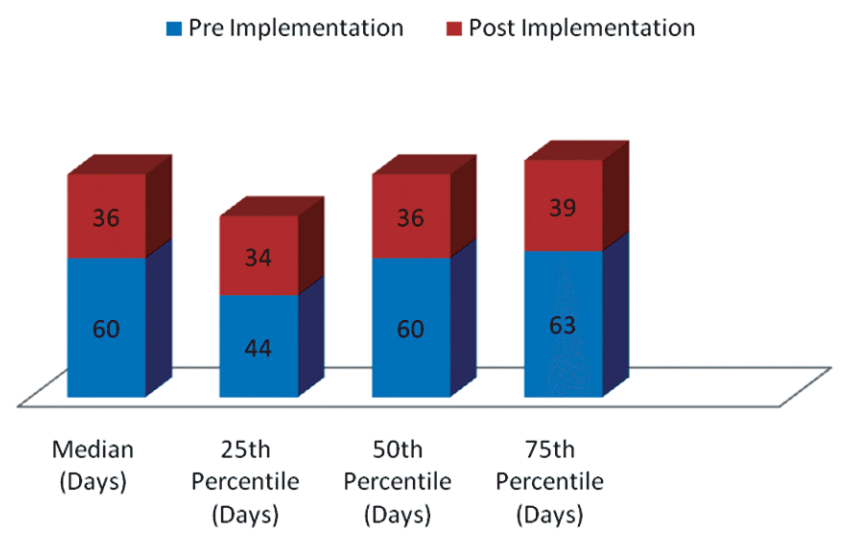

Figure 2: Median number of days and interquartile ranges patient took to be decannulated before and after the implementation of trachesotomy care taskforce.

shorter in the post-implementation group compared to the pre-implementation group $(34.1 \pm 3.6$ vs. $58.0 \pm 11$ days, $P<.001)$. There were no differences in the average number of days from decannulation to hospital discharge in both groups $(P=.92)$. The most common reason for weaning and decannulation failure was poor neurologic state and $\leq 8$ GCS at the time of assessment, followed by a weak cough reflex and increased tracheal and bronchial secretions in both pre- and post-implementation phases.

Two (4\%) patients in pre- and $1(2 \%)$ in postimplementation group were indentified with subglottic stenosis, during weaning trials, which was confirmed by 
computed tomography of neck and direct inspection of airway with fiber optic bronchoscopy. All three patients with sub glottis stenosis were decannulated during insertion of endotracheal stent. Two (4\%) patients in pre- and $4(8 \%)$ in post-implementation phase required ICU admission for pneumonia and sepsis and they died with tracheostomy tube in the ICU. It is to be noted however that none of the patients who were decannulated required ICU admission or reintubation. There was no post-decannulation mortality in either phase of the study.

As the intensivist lead TCT was responsible for the care of all tracheostomized patients, we were able to follow all 35 patients who failed decannulation in pre-implementation phase. While none of these patients have included in postimplementation data, it is important to note that $16(46 \%)$ patients were successfully decannulated and discharged from the hospital.

\section{Discussion}

Introduction of PCT technique has significantly simplified the care of patients, who require prolonged weaning from mechanical ventilator. Early tracheostomy seems to decrease the duration of mechanical ventilation and length of ICU stay in certain critically ill populations, without effecting the morbidity and mortality $[1,2,4,5,8,9,10]$. Post tracheostomy care goes uninterrupted during the patient's stay in the ICU, but unfortunately there is no consensus regarding who is going to follow these patients after an ICU discharge. Most ICUs have no dedicated outside services and many primary care physician and surgeons may not feel comfortable about making decisions about closure and downsizing of tracheostomy and/or decannulation. This may further delay the process of weaning and decannulation limiting discharge of such patients to community.

Our study suggests that a specialized tracheostomy team that assesses the patients post ICU discharge may lead to an increase in the number of decannulations, shorten time of decannulation post ICU discharge and thereby lead to an early hospital discharge. The results of our study clearly support the earlier work published by Tobin et al. [12], in which multidisciplinary team approach for tracheostomized patients in the ward improved the outcome. Tobin et al. also demonstrated that shorter decannulation time and length of hospital stay can be achieved by introducing a multidisciplinary tracheostomy team. The author suggested that the improved outcome was multifactorial and one of the major factors was regular review by an experienced team [12].

Having the identical baseline characteristics of patients in both groups of our study as well as the use of the same weaning protocol throughout the study period, the increased number of decannulations and the reduced number of days with tracheostomy tube post ICU discharges can most likely be explained by the committed team of experts lead by an intensivist with regular and close follow ups of tracheostomized patients. Successful decannulations of $46 \%$ patients who failed weaning and decannulation in pre-implementation phase during the post-implementation phase reiterates the importance of having a dedicated tracheostomy team. This change in practice also helped us in creating space for a smoother patients flow from ICU to wards and opened up more critical care beds for critically ill patients.

The majority of our patients were expatriates working in Dubai while their families were living in their country of origin. Most of them were manual workers and were unable to resume their jobs due to their functional status at the time of discharge. Repatriation of these patients with a tracheostomy tube is not safe secondary to inadequate resources in their country of origin. Unlike Western countries, there are no chronic care or rehabilitation centers available in UAE, where such patients can be transferred. In the study by Tobin [12], $70 \%$ of the decannulated patients were discharged to rehabilitation center, another hospital or aged care center. There were total of 49 patients in our study who were repatriated safely to their home countries during the study period.

Studies have found that tracheostomized patients are high risk patients, when the tube is left in place at ICU discharge, mortality ranges from $17 \%$ to $39 \%$, which was much higher when compared to patients who were decannulated in the ICU prior to discharge [3,6,7]. None of these studies, which looked into the mortality of this high risk group of patients, have mentioned an organized tracheostomy care team following these patients post ICU discharge, which may explain high mortality rate in the studied population. These high risk patients need close follow up from the physicians, who are well qualified with necessary expertise in managing them and familiar with the possible complications that may happen. As most of these procedures are performed by the intensivist, it is unethical to expect other services like ear, nose, and throat physician or primary service to follow and manage these patients. However, most of the ICUs have no multidisciplinary tracheostomy team to follow these patients regularly in the ward post ICU discharge.

The other area which needs standardization is the process or algorithm for weaning and decannulation of these patients. Specific weaning and decannulation strategies are frequently institution-dependent. Some consider it once the patient has had the tracheostomy tube plugged for 48 hours or more, whereas others consider it once a speaking valve is tolerated [11]. We found our standardized protocol for the weaning and decannulation very useful. The screening criteria assisted identification of patients ready for a weaning trial. It also prevents unsafe prolongation of weaning, when patients are not medically fit for the trial. By using this 
protocol we were also able to identify three patients who failed decannulation due to subglottic stenosis. This posttracheostomy complication was appropriately managed by inserting tracheal stent, decannulation, and repatriation.

The mortality in our study group was only $4 \%$ and $8 \%$ in pre- and post-implementation phases, which is much lower than the previous studies. Despite the fact that there was no organized team during pre-implementation phase, our finding of lower mortality in pre-implementation phase may be due to the hospital policy. Our institution policy enforces that all tracheostomy related issues from routine change to acute obstruction and cuff related problems must be looked after by an intensivist. In addition, our ICU team also leads cardiac arrest calls and rapid response team in the hospital.

Some of the strengths and weaknesses of the present study are worth mentioning. We had no exclusion criteria and as a result we mimic the actual practice including all post ICU discharges with tracheostomy tube in place including patients with poor neurologic recovery. We also followed patients who failed decannulation in preimplementation phase, and successfully decannulated almost $50 \%$ of them supporting our hypothesis that dedicated tracheostomy care teams can improve patient care. We did not observe any complication post decannulation and there was no increase in mortality related to the intervention. An important limitation of our study was retrospective data collection during pre-implementation phase and no-long term follow up on patients, who were discharged and repatriated to their country of origin.

As a result of this study, the hospital administration made a policy for regular follow up of all patients who are discharged from the ICU with tracheostomy tube by a TCT. The nursing department has also approved a new hospital-based guideline for nursing practice for managing adult tracheostomized patients.

\section{Conclusion}

Introduction of a structured multidisciplinary tracheostomy care team was associated with a significant increase in the number of decannulations, hospital discharges, and repatriation and less hospital days post ICU discharge.

\section{References}

[1] Y. Arabi, S. Haddad, N. Shirawi, and A. Al Shimemeri, Early tracheostomy in intensive care trauma patients improves resource utilization: a cohort study and literature review, Crit Care, 8 (2004), R347-R352.

[2] M. A. Bouderka, B. Fakhir, A. Bouaggad, B. Hmamouchi, D. Hamoudi, and A. Harti, Early tracheostomy versus prolonged endotracheal intubation in severe head injury, J Trauma, 57 (2004), 251-254.

[3] C. Clec'h, C. Alberti, F. Vincent, M. Garrouste-Orgeas, A. de Lassence, D. Toledano, et al., Tracheostomy does not improve the outcome of patients requiring prolonged mechanical ventilation: a propensity analysis, Crit Care Med, 35 (2007), 132-138
[4] C. M. Dunham and C. LaMonica, Prolonged tracheal intubation in the trauma patient, J Trauma, 24 (1984), 120-124.

[5] C. M. Dunham and K. J. Ransom, Assessment of early tracheostomy in trauma patients: a systematic review and metaanalysis, Am Surg, 72 (2006), 276-281.

[6] F. Frutos-Vivar, A. Esteban, C. Apezteguía, A. Anzueto, P. Nightingale, M. González, et al., Outcome of mechanically ventilated patients who require a tracheostomy, Crit Care Med, 33 (2005), 290-298.

[7] G. H. Martinez, R. Fernandez, M. S. Casado, R. Cuena, P. LopezReina, S. Zamora, et al., Tracheostomy tube in place at intensive care unit discharge is associated with increased ward mortality, Respir Care, 54 (2009), 1644-1652.

[8] J. L. Rodriguez, S. M. Steinberg, F. A. Luchetti, K. J. Gibbons, P. A. Taheri, and L. M. Flint, Early tracheostomy for primary airway management in the surgical critical care setting, Surgery, 108 (1990), 655-659.

[9] M. J. Rumbak, M. Newton, T. Truncale, S. W. Schwartz, J. W. Adams, and P. B. Hazard, A prospective, randomized, study comparing early percutaneous dilational tracheotomy to prolonged translaryngeal intubation (delayed tracheotomy) in critically ill medical patients, Crit Care Med, 32 (2004), 16891694.

[10] J. R. Saffle, S. E. Morris, and L. Edelman, Early tracheostomy does not improve outcome in burn patients, J Burn Care Rehabil, 23 (2002), 431-438.

[11] R. E. St John and J. F. Malen, Contemporary issues in adult tracheostomy management, Crit Care Nurs Clin North Am, 16 (2004), 413-430.

[12] A. E. Tobin and J. D. Santamaria, An intensivist-led tracheostomy review team is associated with shorter decannulation time and length of stay: a prospective cohort study, Crit Care, 12 (2008), R48. 